\title{
Deep-level defects in CdSe/ZnSe QDs and giant anti-Stokes photoluminescence
}

\author{
M.Ya. Valakh, Yu.G. Sadofyev*, N.O. Korsunska, G.N. Semenova, \\ V.V. Strelchuk, L.V. Borkovska, M.V. Vuychik, M. Sharibaev \\ Institute of Semiconductor Physics, NAS of Ukraine, 45 prospect Nauky, 03028 Kyiv, Ukraine \\ Phone: +38-044-2658550; fax: +38-044-2658342; e-mail: valakh@semicond.kiev.ua \\ *P.N. Lebedev Physical Institute, RAS, 117927 Moscow, Russia
}

\begin{abstract}
CdSe} / \mathrm{ZnSe}$ structures with a quantum dot extrinsic photoluminescence band related to the defects that contain vacancies in cation sublattice has been investigated. It is shown that such defects can be localized in different parts of heterostructure (inside $\mathrm{ZnSe}$ barrier and cap layers, $\mathrm{Zn}_{1-\mathrm{x}} \mathrm{Cd}_{\mathrm{x}} \mathrm{Se}$ wetting layer and at quantum dot heterointerface) and their localization depends on the preparation regimes and parameters of investigated structures. It is shown that defect level follows the heavy-hole related level. An intense anti-Stokes photoluminescence of quantum dots has been found. Two-step excitation mechanism of the anti-Stokes photoluminescence through the local states of investigated defects localized on the quantum dot interface is proposed.
\end{abstract}

Keywords: quantum dot, deep-level defects, anti-Stokes photoluminescence.

Paper received 18.07.02; accepted for publication 10.12.02.

\section{Introduction}

The CdSe/ZnSe-based quantum dot (QD) nanostructures are investigated widely because of the possible application for fabrication of blue-green lasers. In these systems a high concentration of cation vacancies is known to be generated during the growth processes. It can lead to the appearance of recombination channels that limit an efficiency of the QDs emission. On the other hand, lifetime of the carriers captured by such defects can be essentially higher and therefore these defects can be effective in the processes of anti-Stokes photoluminescence excitation [1]. In this work the influence of the defects including cation vacancies on the optical properties of heterostructures containing $\mathrm{CdSe} / \mathrm{ZnSe}$ quantum dots has been studied. Anti-Stokes photoluminescence of quantum dots has been investigated.

\section{Experiment and results}

The studied structures were grown on GaAs(100) by MBE and contained either single sheet or twelve vertically stacked Cd-rich sheets separated by $\sim 18$ (or 50) $\mathrm{nm} \mathrm{ZnSe}$ spacer. All samples were grown on $200 \mathrm{~nm}$ thick $\mathrm{ZnSe}$ buffer layer and were capped by $100 \mathrm{~nm} \mathrm{ZnSe} \mathrm{layer.} \mathrm{ZnSe}$ buffer and ZnSe barriers were grown at the temperatures of $280^{\circ} \mathrm{C}$ and $230^{\circ} \mathrm{C}$, correspondingly. After the deposition of each CdSe layer the $\mathrm{Cd}$ beam was blocked and the structure was heated up to $340{ }^{\circ} \mathrm{C}$ and then cooled down to $230^{\circ} \mathrm{C}$ under Se flux. Time duration of both the temperature increase and decrease was 4 minutes. After such a procedure next $\mathrm{ZnSe}$ barrier or top layer was grown. The RHEED method was used to in situ control of threedimensional island formation. Two series of multistack structures were grown. The samples of series 1 as well as single-sheet ones were grown at VI/II group beam pressure ratio of $3: 1$ while the samples of series 2 - at 5:1 ratio. In the samples of series 1 the nominal thickness of CdSe layer were 3.5 monolayer (ML) and ZnSe barrier thickness was $50 \mathrm{~nm}$. In the samples of series 2 the nominal thickness of CdSe layers were 2.1-3.5 ML and the thickness of $\mathrm{ZnSe}$ barriers were $18 \mathrm{~nm}$.

The photoluminescence (PL) and photoluminescence excitation (PLE) spectra were measured in the temperature range $4.2-300 \mathrm{~K}$. Photoluminescence was excited by the discrete lines of $\mathrm{Ar}^{+}$or $\mathrm{N}_{2}$-laser. For PLE measurements a glow lamp was used.

The Fig.1 shows the PL spectra (curves 1 ) at $T=77 \mathrm{~K}$ of the single-sheet $(a)$ and multistack $(b, c)$ samples of series $1(b)$ and $2(c)$. In PL spectra of all the structures an intense band $I_{Q D}$ at $2.33-2.69 \mathrm{eV}$ with FWHM of 
M.Ya. Valakh et al.: Deep-level defects in CdSe/ZnSe QDs and giant anti-Stokes photoluminescence
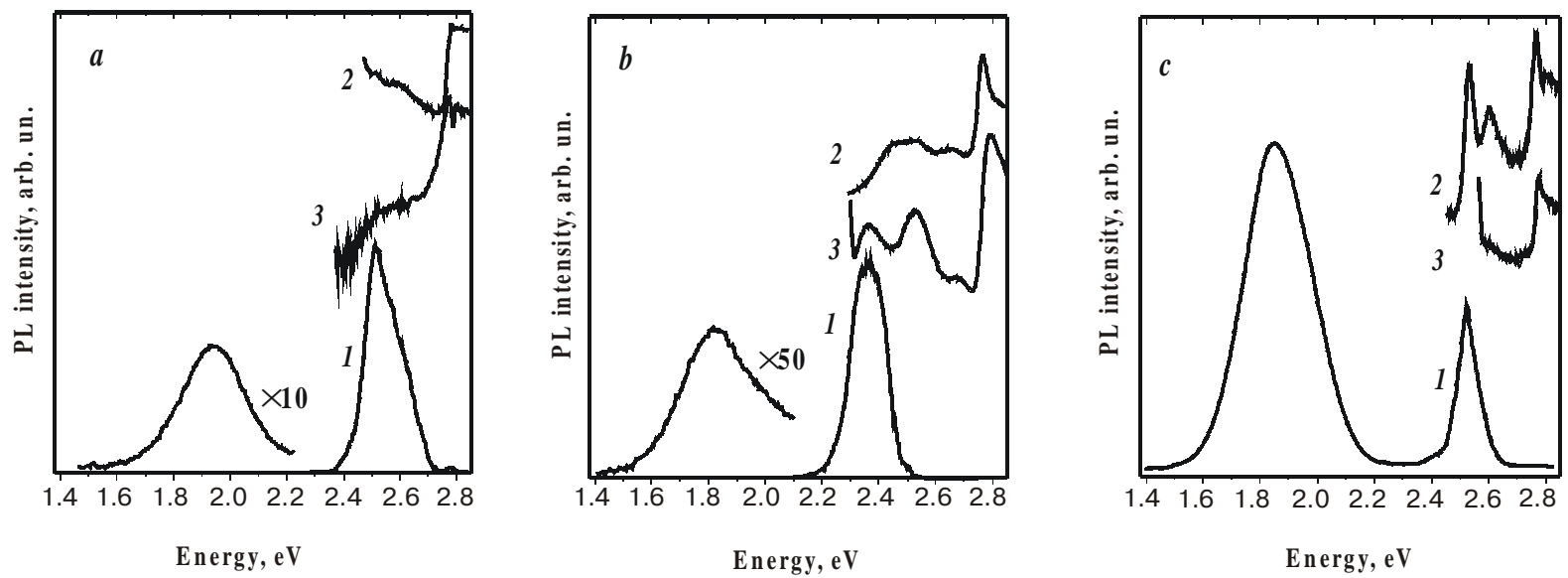

Fig. 1. PL (curve 1) and PL excitation spectra at $T=77 \mathrm{~K}$ of $I_{D}$ (curve 2) and $I_{Q D}$ (curve 3 ) bands of structures contained single sheet Cd-rich dots $(a)$ or twelve vertically stacked Cd-rich dots of series $1(b)$ and $2(c)$. The nominal thickness of CdSe layers is $3.5 \mathrm{ML}$, $\mathrm{ZnSe}$ barrier thickness is $50 \mathrm{~nm}(b)$ and $18 \mathrm{~nm}(c) . \lambda_{\text {exc }}=440 \mathrm{~nm}$ (curve 1).

$28-115 \mathrm{meV}$ and broad defect-related band $I_{D}$ at 1.82 $1.99 \mathrm{eV}$ are observed. Increase of the nominal thickness of CdSe layer in multistack samples of series 2 results in the shift of both $I_{O D}$ and $I_{D}$ band maximum positions to the low-energy side of the spectral range (Fig.1, $c$ ). Spectral position of $I_{Q D}$ band is in agreement with the results obtained for the structures grown by the similar methods [2] where such band was interpreted as radiative recombination of the e-hh exciton in quantum dots.

The excitation spectra of $I_{D}$ (curve 2 ) and $I_{Q D}$ bands (curves 3) are also presented in Fig.1. In PL excitation spectra of $I_{O D}$ band (Fig.1, curve 3), besides the features corresponding to the light absorption in $\mathrm{ZnSe}$-barrier $(\sim 2.8 \mathrm{eV})$, one can see the double band corresponding to heavy-hole and light-hole transitions in QDs. All these features are present in PL excitation spectra of $I_{D}$ band (Fig.1, curve 2) of multistack samples of series 2 (Fig.1c) and single-sheet ones (Fig.1a). However, for single-sheet structures, the features corresponding to light absorption in QDs are not pronounced so clear. In PL excitation spectra of $I_{D}$ band of the series 1 structures (Fig. $1 b$, curve 2) only the features corresponding to light absorption in $\mathrm{ZnSe}$ barrier and $\mathrm{Zn}_{1-\mathrm{x}} \mathrm{Cd}_{\mathrm{x}} \mathrm{Se}$ wetting layer (a shoulder in $2.4-2.7 \mathrm{eV}$ range) are observed. It has been found that intensity, shape and spectral position of the maximum of $I_{D}$ band depend essentially on the preparation regimes and parameters of investigated structures (number and nominal thicknesses of CdSe layers). In particular, in multistack samples an intensity of $I_{D}$ band in the samples of series 2 is one order greater than intensity of such band in the samples of series 1. In multistack samples of series 1 (Fig. $1 b$ ) the $I_{D}$ band consists of two components, the spectral position of its high-energy shoulder is close to the spectral positions of the emission bands connected with the $\mathrm{V}_{\mathrm{Zn}}$-D defects observed in $\mathrm{ZnSe}$ layers [3].

Spectral position of $I_{D}$ band has been found to depend on the energy of excitation quanta. The $I_{D}$ band maximum position shifts to low-energy region under chang- ing the excitation energy from $2.8 \mathrm{eV}$ to $2.3-2.7 \mathrm{eV}$ region. Nevertheless, for multistack structures this shift is small $(\sim 10 \mathrm{meV})$.

It should be noted that for all investigated samples a relation between $I_{D}$ and $I_{Q D}$ band maximum positions is observed. However, a clear correlation is found only for the samples of series 2, for which the dependence of $I_{D}$ band maximum position $\left(h v_{D}\right)$ on the $I_{Q D}$ band maximum position $\left(h v_{Q D}\right)$ is linear (see Fig. 2, solid circles). The point corresponding to the structures of series 1 does not deviate from this dependence, if the low-energy edge of $I_{D}$ band excitation spectra corresponding to the light absorption in wetting layer is taken instead of $I_{Q D}$ band maximum position. The point corresponding to the single-sheet sample is also presented in Fig.2 (open circle).

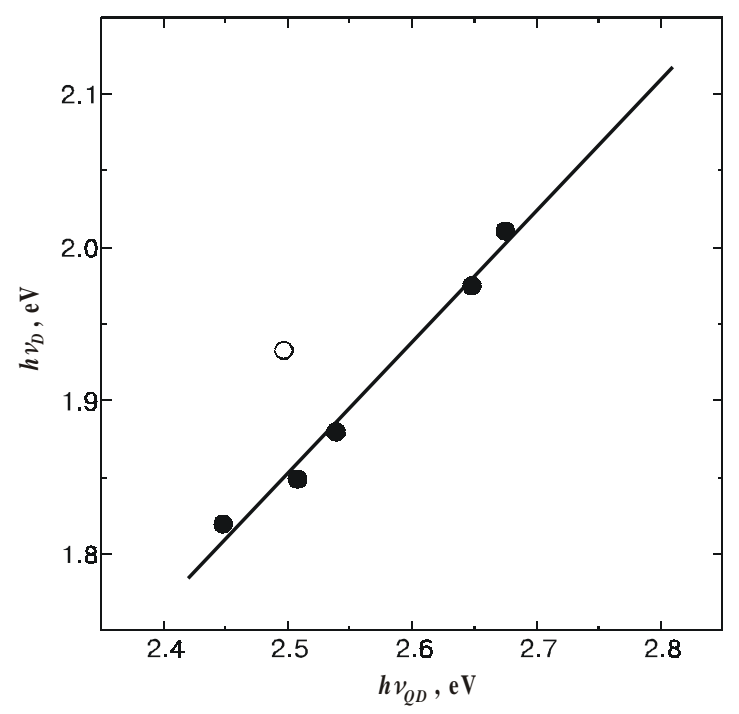

Fig. 2. Dependence of $I_{D}$ band maximum position $\left(h v_{D}\right)$ on the $I_{Q D}$ band maximum position $\left(h v_{Q D}\right)$ for multistack samples (solid circles) and single-sheet structure (open circle) at $T=77 \mathrm{~K}$, $\lambda_{\text {exc }}=337 \mathrm{~nm}$. 


\section{M.Ya. Valakh et al.: Deep-level defects in CdSe/ZnSe QDs and giant anti-Stokes photoluminescence}

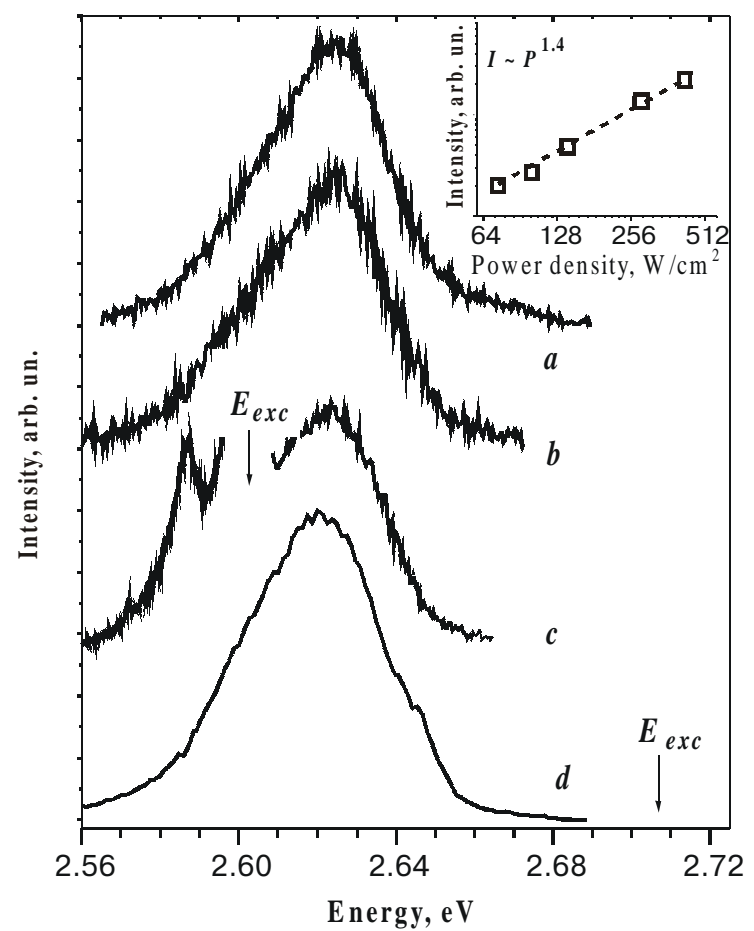

Fig. 3. Evolution of QD PL spectra at different values of excitation energy: $a-2.707 ; b-2,602 ; c-2.54 ; d-2.41 \mathrm{eV}$.

The Fig. 3 shows the PL spectra of the sample of series 2 measured at $T=5 \mathrm{~K}$ under excitation on highenergy $\left(E_{\text {exc }}=2.71 \mathrm{eV}\right)$ and low-energy $\left(E_{\text {exc }}=2.54\right.$ and $2.41 \mathrm{eV})$ sides of $I_{O D}$ band as well as under resonant excitation $\left(E_{\text {exc }}=2.6 \mathrm{eV}\right)$. It is seen that anti-Stokes PL band is somewhat shifted to high-energy region in comparison with Stokes band and has a smaller (by 3 to $5 \mathrm{meV}$ ) value of FWHM. The dependence of anti-Stokes PL on the excitation density was superlinear with the factor 1.4 (insert in Fig. 3). For the Stokes PL this dependence was linear. An efficiency of anti-Stokes PL was a few percents of the Stokes PL one. Under resonant excitation in QD PL band, the new band at $2.586 \mathrm{eV}$ and with FWHM of $5 \mathrm{meV}$ appears in the Stokes region. This band can be defined as an exciton recombination of the resonantly excited ensemble of QDs with the definite size and composition.

\section{Discussion}

Since the increase of Se flux pressure results in the increase of $\mathrm{I}_{\mathrm{D}}$ band intensity, it can be concluded that this emission is connected with a cation vacancy related defect. Our results have shown also that $I_{D}$ band consists of several PL bands connected with the defects localized in different parts of the heterostructure. Really, on the one hand, in the PL excitation spectra of $I_{D}$ band the characteristic features corresponding to the light absorption in different parts of the structure (ZnSe barriers, wetting layer and quantum dots) are present. On the other hand, the $I_{D}$ band maximum position depends on the region of heterostructure where carriers are generated. Moreover, in some samples the components of $I_{D}$ band are resolved. For example, in multistack structures of series 1 (Fig. $1 b$ ), two components are seen in the $I_{D}$ band: the maximum and well resolved high-energy shoulder. It is naturally to relate the high-energy component with the centers localized in ZnSe barrier and the low-energy one - with the centers localized in the wetting layer. It is proved by the fact that only ZnSe barriers and wetting layer are observed in the excitation spectra of the $I_{D}$ band. It should be noted that absence of the emission centers on the quantum dot interface in the samples of series 1 can be caused by larger sizes of these dots. Appearance of extended defects that are the sink for point defects is expected near such dots [3]. The bigger size of these dots in comparison with samples of series 2 that have the same 3.5 ML CdSe inserts is confirmed by more low-energy position of its $I_{Q D}$ band.

At the same time, in the samples of series 2, a considerable amount of defects is present on the quantum dot heterointerface (excitation through the quantum dots is effective). Observed insignificant difference in the $I_{D}$ band maximum positions under excitation through the $\mathrm{ZnSe}$ barrier or QDs testifies to the main contribution into the emission of the defects localized at QD interface. Correlation of the $I_{D}$ and $I_{O D}$ band maximum positions means that the position of defect level on the quantum dot interface follows the quantized heavy-hole level of QDs. For the centers localized in the wetting layer (samples of series 1), the defect level follows the quantized heavy-hole level of the wetting layer. Approximation of the linear dependence presented in Fig. 2 to the bulk $\mathrm{ZnSe}$ gives the $I_{D}$ band maximum position $\sim 2.1 \mathrm{eV}$. Such emission band was really observed by us in ZnSe buffer layers [3]. The observed deviation of $I_{D}$ band maximum position for single QD sheet samples from the linear dependence presented in Fig. 2 can be caused by the significant contribution of the emission from the defects localized in $\mathrm{ZnSe}$ because of relatively smaller amounts of CdSe in such structures.

The presence of significant quantity of cation vacancy related defects in investigated samples could cause observed intense anti-Stokes PL. In our case, the excitation mechanism of giant anti-Stokes PL can be in the best way explained by the two-step excitation process through the local levels of defects localized in Cd-rich layers. Really: i) in investigated samples these defects are present at QD heterointerface; ii) energy levels of such defects are enough deep. It is known that electron lifetime on the centers involving cation vacancy is sufficiently high $\left(\sim 10^{-3} \mathrm{c}\right)[4]$; iii) in the case of two-step excitation mechanism of the anti-Stokes PL, the intensity dependence on the excitation density could be characterized by the power law with the factor 2 and less, that is in agreement with experimental data obtained.

In conclusion, it should be noted that smaller value of FWHM as well as the shift of the anti-Stokes PL band maximum position in the high-energy region in comparison with the Stokes band can be explained in assumption 
M.Ya. Valakh et al.: Deep-level defects in CdSe/ZnSe QDs and giant anti-Stokes photoluminescence

that the quantity of emission centers localized at QD interface decreases with increasing QD size. This is in agreement with earlier suggestion about the reasons of lack of such centers at QD interface in the samples of series 1.

\section{References}

1. M. Strassburg, M. Kuttler, U.W. Pohl, D. Bimberg, Diffusion of $\mathrm{Cd}, \mathrm{Mg}$ and $\mathrm{Si}$ in $\mathrm{ZnSe}$-based quantum well structures // Thin. sol. films 336, pp.208-212 (1998).
2. M. Rabe, M. Lowish, F. Henneberger, Self-assembled CdSe quantum dots. Formation by thermal activated surface reorganization // J. Cryst. Growth. 184/185, pp.248-253 (1998).

3. D. Litvinov, D. Gerthsen, A. Rosenauer, H. Preis, E. Kurtz and C. Klingshirn, $\mathrm{Cd}$ distribution and defects in single and multilayer quantum dot structures // Phys. stat. sol. (b). 224, pp.147-151 (2001).

4. V.E. Lashkar'ov, A.V. Lubchenko, M.K. Sheynkman, Nonequilibrium processes in photoconductors, Naukova dumka, Kiev (1981). 